\title{
Robert Leonard Carneiro (1927-2020)
}

\section{Philippe Erikson}

\section{OpenEdition}

\section{Journals}

Édition électronique

URL : https://journals.openedition.org/jsa/18841

DOI : 10.4000/jsa.18841

ISSN : 1957-7842

\section{Éditeur}

Société des américanistes

\section{Édition imprimée}

Date de publication : 15 décembre 2020

Pagination : 233-243

ISSN : 0037-9174

\section{Référence électronique}

Philippe Erikson, «Robert Leonard Carneiro (1927-2020)», Journal de la Société des américanistes [En ligne], 106-2 | 2020, mis en ligne le 30 décembre 2020, consulté le 16 septembre 2022. URL : http:// journals.openedition.org/jsa/18841 ; DOI : https://doi.org/10.4000/jsa.18841 


\title{
Robert Leonard Carneiro (1927-2020)
}

\author{
Philippe ERIKSON *
}

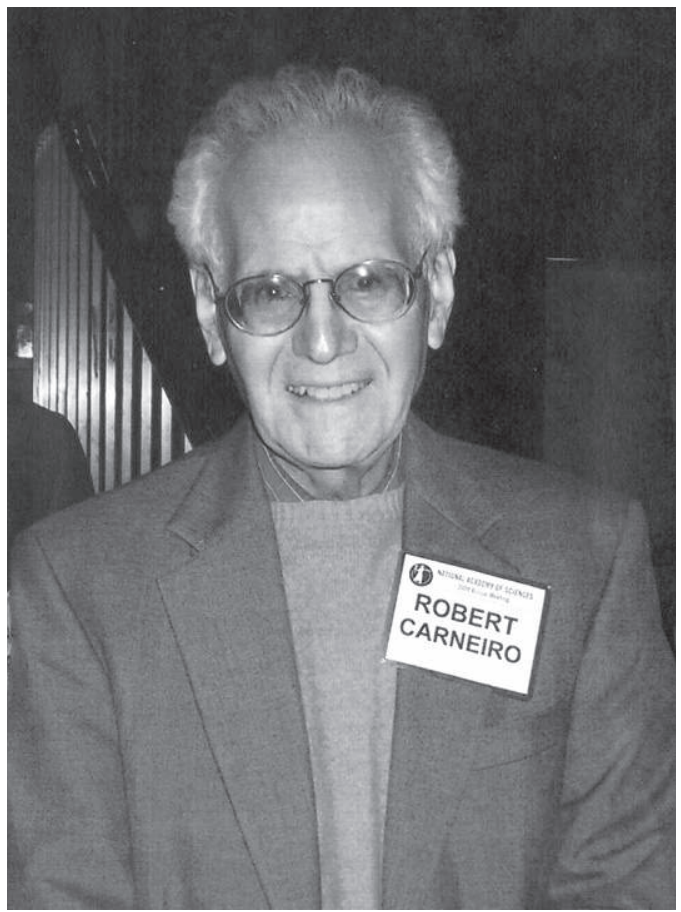

Fig. 1 - Robert Leonard Carneiro

(C) Courtesy of the Division of Anthropology, American Museum of Natural History)

Robert L. Carneiro est décédé le 24 juin 2020, quelques semaines après la célébration de son $93^{\mathrm{e}}$ anniversaire. Il laisse le souvenir d'un pionnier de l'ethnographie amazonienne, d'un anthropologue à l'œuvre féconde et d'un

* Université Paris Nanterre, centre EREA du LESC (UMR 7186) [erikson@parisnanterre.fr]. 
muséographe influent, qui travailla plus d'un demi-siècle dans ce haut-lieu de l'anthropologie nord-américaine qu'est l'American Museum of Natural History (AMNH). « Bob » laisse aussi le souvenir d'un homme austère mais accueillant, qui ouvrait généreusement ses archives aux jeunes chercheurs qui venaient le rencontrer dans le bureau qu'il occupa, de 1957 à 2009, à Central Park West, 79th Street, New York.

Accompagné de sa première épouse, Gertrude Dole ${ }^{1}$, Carneiro fit ses premières armes sur le terrain au Brésil, chez les Kuikuru du Haut-Xingu, en 1953-1954, à une époque où l'américanisme tropical était encore balbutiant. Tandis que « Trudie » s'intéressait surtout à l'organisation sociale, Bob se consacra plus particulièrement à l'anthropologie économique, notamment la productivité des jardins. En résulta une thèse soutenue en 1957 qui démontrait, chiffres à l'appui, l'inanité de l'image misérabiliste que l'on se faisait alors des activités de subsistance des Amérindiens. Les calculs sophistiqués de Carneiro établissaient clairement que, pour ce qui concerne l'agriculture, la capacité de charge de leur environnement dépassait largement le minimum vital nécessaire pour couvrir leurs besoins et que l'extrême mobilité des Amazoniens n'avait donc rien d'une fatalité. Cela peut sembler aujourd'hui trivial mais à l'époque, presque vingt ans avant la vogue des « sociétés d'abondance ${ }^{2}$ », cette révélation eut un retentissement considérable. On peut même dire que les résultats de Carneiro ont réorienté toute la recherche américaniste pour le quart de siècle à venir. Jusqu'au milieu des années 1980, les spécialistes de l'Amazonie se lancèrent à cœur perdu dans un houleux débat sur les protéines, n'ayant de cesse de chercher

1. Sur la carrière de Gertrude Evelyn Dole (1915-2001), on peut consulter les nécrologies exhaustives de Barnes (2003) et de Chernela (2005). L'œuvre de Dole est très complémentaire de celle de Carneiro (e.g. Dole 1960), et l'aurait sans doute été plus encore sans les injonctions machistes des hiérarques de l'université du Michigan. Craignant que son époux ne rédige à sa place, ces derniers ont en effet rejeté son projet de thèse initial sur l'organisation sociale des Kuikuru, lui imposant à la place un travail comparatif sur les terminologies de parenté (Stoll 2020).

2. «Sociétés d'abondance » est l'expression choisie pour rendre l'idée « d'affluent societies » mise à l'honneur par le best-seller de Sahlins (1976 [1974]). L'étude de l'influence de Carneiro sur ce dernier reste encore à faire. Notons simplement qu'ils ont fréquenté les mêmes universités (Michigan, Columbia), qu'ils ont tous deux été proches d'Elman Service, et fortement marqués l'un comme l'autre par l'évolutionnisme matérialiste de Leslie White. Sahlins a bien sûr ultérieurement abjuré le matérialisme culturel dans la seconde moitié de sa carrière, tandis que Carneiro, pour sa part, s'est montré d'une constance absolue dans la défense et illustration de l'évolutionnisme. En 2009, c'est tout juste s'il concédait que: « As a graduate student I used to disdain the study of mythology. To me it seemed trivial, and at best peripheral to the solid substance of anthropology. But over the years, especially as I began to do ethnographic field work and to collect myths myself, my attitude toward mythology changed. For one thing, I became more fully aware of - and began to admirethe touches of imagination so much in evidence in the myths of primitive [sic] peoples » (Carneiro 2009, p. 57). 
du côté animal les carences que Carneiro avaient définitivement évacuées, côté végétal $^{3}$. Ceci étant, quoique matérialiste invétéré, Carneiro n'hésitait pas à fustiger l'intransigeance dogmatique des absolutistes du déterminisme environnemental, notamment dans sa variante du « tout protéique ». En témoignent les commentaires publiés dans Current Anthropology, lors de la sortie des travaux incriminés. À Ross, il rappelait par exemple que: "There is always a residue of culture that may never be explainable ecologically. The size of this residue may be debated, but its existence can-not be denied. As cultural ecologists we should content ourselves with a preponderance of triumphs and not demand total subjugation » (Carneiro 1978, p. 20). À la différence de bon nombre de matérialistes culturels qui honnissaient et rejetaient tout de go le " mentalism » de Lévi-Strauss, Carneiro professait pour sa part une grande admiration pour l'étendue de l'érudition de ce dernier.

Carneiro retourna chez les Kuikuru en 1975, seul, pour un bref séjour estival à l'issu duquel il publia quelques articles sur des thématiques inhabituelles pour lui, telles que la mythologie, la sorcellerie et l'eschatologie. Grand admirateur de Herbert Spencer ${ }^{4}$, il ne se départit cependant jamais d'un positivisme clairement assumé et il est remarquable que son article sur la mythologie kuikuru comprenne plus de notes que de texte: cent soixante-dix pour être exact, preuve d'une attention forcenée aux détails concrets (Carneiro 1989). Imperturbable dans ses convictions, ni le mouvement post-moderne ni le tournant ontologique n'eurent la moindre incidence sur son parti-pris évolutionniste, matérialiste et scientiste (Carneiro 1995, 2004). Carneiro aura toujours été obstinément méthodique et radicalement rigoureux même si, nous y reviendrons, ses spéculations sur l'histoire globale de l'humanité supposaient une bonne dose d'imagination.

En 1960-1961, toujours accompagné de Gertrude Dole, Robert L. Carneiro se rendit chez les Amahuaca d'Amazonie péruvienne. Le choix de ce second terrain avait été dicté par son recrutement à l'AMNH, dont 1'essentiel des collections amazoniennes provenaient du piémont andin. Une connaissance de première main de la région était donc requise, d'autant que le précédent conservateur pour l'Amérique du sud, l'andiniste Harry Tschopik, avait monté une exposition intitulée Men of the Montaña, que Carneiro avait pour mission de faire évoluer en exposition permanente (Tschopik 1952; Carneiro 2019).

3. Deux recueils d'articles marquants sur l'Amazonie sont parus au début des années 1970. L'article pionnier de Carneiro sur l'agriculture sur brûlis est le seul texte à figurer dans l'un comme dans l'autre de ces manuels qui ont influencé plusieurs générations d'étudiants (Carneiro 1961, repris, entre autres, par Gross [1973] et par Lyon [1974]). On peut voir là le signe incontestable de l'importance de ses premiers travaux, d'autant que les orientations théoriques de Gross et de Lyon étaient pour le moins contrastées.

4. Son admiration sans bornes pour Spencer incita Carneiro à lancer le site: https://www. amnh.org/research/research-library/search/research-guides/herbert-spencer-cyclopedia, consulté le 15/12/2020. 
L'exposition supposément temporaire, à laquelle il s'agissait de donner plus d'envergure, dura en fait plusieurs décennies, jusqu'en 1976. Dans l'intervalle, Carneiro avait pu se familiariser personnellement avec l'Amazonie péruvienne et, en parallèle de son travail de conservateur, se consacrer à l'enseignement et la recherche.

Sans doute le terrain dans la Montaña aura-t-il été moins productif que celui réalisé dans le Xingu, ne serait-ce qu'en raison de sa moindre durée. Il aura néanmoins un impact essentiel auprès du grand public, d'une part à cause de l'importance des Amahuaca dans la scénographie de la galerie sud-américaine de l'AMNH, d'autre part, en raison de la contribution de Dole et Carneiro à l'élaboration de Farewell to Eden (Huxley et Capa 1964), que l'on peut sans doute considérer comme l'un des plus remarquables livres de vulgarisation jamais publié sur un peuple d'Amazonie. Il faut dire qu'il aura été élaboré par une équipe de choc. Outre la contribution d'un des plus grands noms de la photographie du $\mathrm{xx}^{\mathrm{e}}$ siècle (Cornell Capa) et d'un auteur au nom prestigieux $\left(\right.$ Matthew Huxley ${ }^{5}$ ), l'ouvrage a en effet bénéficié des informations détaillées fournies par les missionnaires du SIL, Robert et Delores Russell, relayées par Dole et Carneiro. Ce dernier avait largement préparé le terrain en amont, bombardant de questions les missionnaires qui connaissaient les Amahuaca de longue date (Russell 1962). Lors de notre première rencontre - un quart de siècle plus tard - Carneiro, dont la ténacité ne se démentit jamais, me montra d'ailleurs une boîte contenant plus de 500 fiches méthodiquement annotées. Il s'agissait de toutes les questions qu'il lui restait à poser aux Amahuaca, et qu'il m'aurait volontiers confiées si l'envie me venait un jour d'aller les interroger. Une vingtaine d'années plus tard, c'est un autre jeune collègue, Christopher Hewlett, qui se chargea de poursuivre l'enquête ${ }^{6}$.

En 1975, dans le cadre d'un projet dirigé par Napoleon Chagnon, Carneiro eut l'occasion de séjourner un mois, en compagnie de Kenneth Good, chez les

5. Bien que sa carrière universitaire ait été essentiellement consacrée à l'épidémiologie, Matthew Huxley - fils de l'écrivain Aldous Huxley et cousin de l'ethnologue Francis Huxley (1956) - était lui-même anthropologue. Outre un père illustre, il pouvait aussi se targuer d'avoir travaillé avec Margaret Mead, ce qui ne pouvait pas nuire à la promotion du livre pour la préparation duquel il passa trois mois au Pérou. Une partie des photos publiées dans l'ouvrage ont été prises par Gertrude Dole, bien que la majorité soit l'œuvre de Cornell Capa, professionnel mondialement connu, entre autres, pour sa spectaculaire couverture de la Seconde Guerre mondiale.

6. À l'issue de son doctorat Hewlett créa, au cœur même du territoire amahuaca, la Casa de Cultura Indígena de Nuevo San Martin, Rio Inuya, centre culturel ayant vocation à restituer les archives, sous forme numérique, à la population source (Hewlett 2013, 2016). Carneiro a fortement soutenu cette entreprise, en permettant à Chris Hewlett de devenir research associate de l'AMNH, avant d'être embauché à l'université du Maryland où il codirige actuellement le Center for Research and Collaboration in the Indigenous Americas (CRACIA, http://www.cracia.org/). 
Yanomamö du Venezuela. Au cours de ce troisième terrain, il concentra son intérêt sur les haches de pierre, dont il restait encore quelques spécimens chez ses hôtes, qui firent une démonstration de leur utilisation traditionnelle. Fasciné par les technologies précolombiennes, Carneiro publia, entre 1969 et 1992, six articles sur l'utilisation des haches de pierre chez les Amahuaca, les Héta (Xetá), les Yanomami et même les Tasaday (des Philippines). Il s'intéressa également aux presses à manioc oblongues (les « couleuvres» ou tipiti) auxquelles il consacra deux articles en 2000 et 2007 . Hormis cela, il est surprenant que la culture matérielle occupe une place si restreinte dans l'œuvre de ce conservateur de musée, qui plus est matérialiste convaincu, qu'était Carneiro. Sauf erreur de ma part, la notion de chaîne opératoire n'est par exemple discutée dans aucun de ses travaux, pas plus que ne l'est l'armement, malgré le grand intérêt de Carneiro pour la chasse et la guerre. (Une autre particularité de sa bibliographie, pourtant abondante, est la faible place qu'y tiennent les publications extra-états-uniennes: quoique fier de ses origines cubaines, Carneiro ne s'est jamais beaucoup préoccupé de publier dans des langues autres que l'anglais).

Carneiro avait une connaissance approfondie de la culture matérielle amérindienne, et adorait les discussions sur la diversité des techniques de tressage/ tissage des hamacs ou sur la question des origines des hameçons en os dans les Amériques (post-colombiennes selon lui, en tout cas pour 1'Amazonie)... Cependant, il ne publiait guère sur ces thématiques, préférant consacrer l'essentiel de sa production scientifique, et ce dès le début des années 1960, à des questions d'ordre épistémologique ou à l'anthropologie générale, voire l'histoire de l'humanité. Tout en ne jurant que par la concrétude ${ }^{7}$, Carneiro n'en avait pas moins le sens de la spéculation à large spectre. Plutôt qu'avec les ethnologues, c'est avec les historiens des idées, les préhistoriens et les archéologues qu'il débattait et, bien souvent, cosignait ses textes. D'ailleurs, moins de la moitié de son œuvre porte sur des questions proprement américanistes, le reste étant consacré à des sujets comme l'analyse l'œuvre de Spencer, les théories de l'évolution, l'écologie culturelle, l'impact du milieu sur la démographie antique ou encore la transformation des systèmes politiques après l'avènement du néolithique. Un coup d'œil rapide sur internet suffit d'ailleurs à se convaincre que Carneiro est surtout connu pour ses réflexions sur la question de l'origine de l'État, et notamment sa fameuse circumscription theory selon laquelle seules des contraintes environnementales susceptibles d'obliger les gens à demeurer sur place plutôt qu'à se disperser pouvaient expliquer le passage d'une organisation en bande ou tribu à une organisation en chefferie,

7. En entendant la longue énumération de tout ce qui aurait pu encore être rajouté à l'article que, selon ses recommandations, j'avais soumis à la revue de l'AMNH (Erikson 1990), Carneiro répliqua simplement: "There is already more than enough symbolism in your paper $[\ldots] »$. 
puis en État (Carneiro 1970, 2012). Remarquons au passage que l'on pourrait presque considérer cette théorie (qui est loin de faire l'unanimité) comme le prolongement symétrique et inverse des premières conclusions de Carneiro sur la capacité de charge supposément illimitée du territoire des Kuikuru. Relevons aussi qu'une réflexion sur les sociétés andines tient une place prépondérante dans le développement de la circumscription theory, ce qui n'est sans doute pas sans rapport avec le fait que Carneiro vivait entouré d'andinistes, notamment sa seconde épouse, Barbara Bode ${ }^{8}$ et, bien sûr, son collègue Craig Morris ${ }^{9}$ avec qui il collabora étroitement pour la réfection du South American Exhibit Hall de l'AMNH.

En plus du monde académique, Carneiro marqua en effet d'une empreinte durable celui de la scénographie américaniste. Avec sa comparse Laila Williamson (pour le volet ethnologique) et son homologue Craig Morris (pour le volet archéologique), il consacra une bonne décennie de sa très longue carrière de conservateur à rénover la section sud-américaine de l'AMNH, pour aboutir en 1989 à l'inauguration du Hall of South American Peoples ${ }^{10}$. Le défi était de taille puisqu'il fallait se montrer digne des sections adjacentes, et notamment celles consacrées à l'Amérique du nord et à l'Océanie, respectivement marquées du sceau de rien moins que Franz Boas et Margaret Mead. Le récit de cette aventure épique a été minutieusement relatée dans le tout dernier ouvrage de Carneiro, paru un an avant son décès. Il a malheureusement été imprimé à compte d'auteur, truffé de coquilles, sans iconographie et en caractères immenses (Bob ayant toujours été très myope) ; le livre n'en regorge pas moins d'informations précieuses sur les conditions d'obtention de certaines pièces ainsi, surtout, que sur les enjeux scientifiques, politiques, éthiques et techniques inhérents au montage d'une exposition de cette envergure. Cet ouvrage - dont, la couverture reprend, en l'inversant, celle de Tschopik (Figure 2) - aborde des questions parfois inattendues (du moins pour les ethnologues), telles l'élaboration des mannequins, l'amélioration des vitrines Hahn importées d'Allemagne ou encore, la meilleure manière de présenter un guerrier kayapo brandissant une massue sans que le public ne pense qu'il s'agit d'un joueur de base-ball.

8. Américaniste renommée, Barbara Bode est notamment l'auteure d'une contribution majeure aux disaster studies (1989), avec une étude sur la chute d'un glacier dans les Andes péruviennes, à la suite d'un tremblement de terre.

9. Sur le partenariat avec Craig Morris (1939-2006) au sein du AMNH, on peut se référer à Carneiro et Marcus (2006), Thomas (2007) et Carneiro (2019).

10. Le long délai entre le début et l'aboutissement du projet s'explique par la grave crise financière qui affecta la ville de New York du milieu des années 1960 à la fin des années 1970. Quoique l'AMNH, établissement privé, ait disposé de capitaux suffisants, les bâtiments appartiennent en effet à la municipalité... Sans doute ce concours de circonstances aura-t-il permis à Carneiro de consacrer tant d'énergie à l'anthropologie théorique et à l'épistémologie, comme en atteste la prépondérance de ces thématiques dans sa liste de publications. 

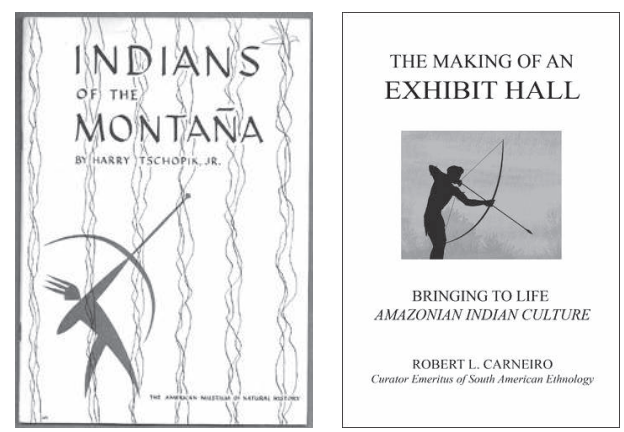

Fig. 2 - Les couvertures de Tschopik (1952) et Carneiro (2019)

(C) Courtesy of the Division of Anthropology, American Museum of Natural History)

Me revient, en écrivant ces lignes, le souvenir d'une discussion avec Bob lors d'une visite privée de la préfiguration de la galerie (à laquelle mon épouse et moi avions contribué avec des photos de Matis). Avec la spontanéité de la jeunesse et sans doute un certain manque de tact, je lui fis remarquer que, sans trop savoir expliquer pourquoi, je trouvais les mannequins « trop réalistes ». Cela laissa mon interlocuteur pantois : comment peut-on être « trop réaliste », alors qu'on ne l'est jamais assez! Tout juste concéda-t-il que les orteils des mannequins auraient pu être plus écartés, comme le sont en effet ceux des Amérindiens qui marchent toute leur vie pieds nus. Finalement, avec la courtoisie qui le caractérisait, Carneiro mit fin à cette situation embarrassante en concluant qu'il voyait ce que je voulais dire, même si j'avais du mal à la formuler à l'époque. Trente ans plus tard, son ouvrage apporte un épilogue rétrospectif à ce débat, en nous apprenant que s'il avait certes été particulièrement soucieux de réalisme, le sculpteur chargé de réaliser les œuvres avait cependant utilisé des modèles pour le moins inattendus. Pour représenter une femme wayana plantant du manioc, il avait par exemple fait poser une strip-teaseuse d'origine espagnole et pakistanaise. La ressemblance lui paraissait suffisante (Carneiro 2019, p. 139).

Plutôt que « Hall of South American Peoples », imposé par la direction du musée, Carneiro aurait préféré que son exposition s'intitule « Native cultures of South America ». Transparait là, bien sûr, un avatar muséographique de la querelle des anciens et des modernes: culturalisme versus décolonisation, valorisation des pièces anciennes versus remise en question de l'autorité du discours anthropologique... À cet égard, le fait que Carneiro ait été conservateur (à tous les sens du terme) à une époque où la tendance était plutôt à l'exposition politiquement correcte des casquettes de base-ball qu'à celle des coiffes de plume d'oiseaux en voie d'extinction, a certainement contribué à rééquilibrer l'offre muséographique américaniste sur la côte est des États-Unis. Il est en tout cas frappant de constater le contraste absolu entre 
les philosophies ayant respectivement présidé à la rénovation de l'AMNH, d'une part (Urton 1992), et du National Museum of the American Indian, de l'autre (Mauzé et Rostkowski 2004). Carneiro avait été ébloui par l'exposition des collections de von den Steinen à Berlin, mais dépité que celles de Nordenskiöld, au fameux musée de Göteborg, aient été remisées au profit d'une scénographie plus tournée vers la mondialisation dans le cadre des Världskultur Museerna.

Entre ses débuts sur le terrain en 1953 et sa toute dernière publication en 2019, plus de soixante-cinq ans se sont écoulés. Et même si l'on raccourcit la période en ne considérant que sa date de retraite (en 2009), on contemple une carrière qui aura largement couvert, et même débordé, la seconde moitié du xx siècle. Á cette longévité s'ajoute une constance remarquable dans les thématiques abordées, ainsi qu'une fidélité indéfectible aux orientations théoriques et aux institutions avec lesquelles il s'était associé. La remarque vaut aussi pour le choix des supports de publication, comme en atteste sa bibliographie. Paradoxalement, Carneiro aura été tout à la fois obstinément conservateur et souvent innovant, très attaché à la froideur des raisonnements quantifiables, et néanmoins chaleureux. Dans un article consacré à l'au-delà des Kuikuru, Bob écrivait que les arts (en l'occurrence verbaux) pouvaient aider à envisager plus sereinement l'évanescence du temps et l'implacable finitude de la condition humaine (Carneiro 1976, p. 12). Il est mort paisiblement, entouré de ses proches, en écoutant du Beethoven.

\section{Références citées}

BARnes Monica

2003 « Gertrude Evelyn Dole (1915-2001)», American Anthropologist, 105 (2), p. 484-486.

Bode Barbara

1989 No Bells to Toll. Destruction and Creation in the Andes, Charles Scribner's Sons (Macmillan), New York.

CARNEIRO Robert Leonard

1961 «Slash-and-burn cultivation among the Kuikuru and its implications for cultural development in the Amazon Basin », in Johannes Wilbert (dir.), The Evolution of Horticulture Systems in Native South America. Causes and Consequences, Sociedad de Ciencias Naturales La Salle (Antropológica. Supplement Publication 2), Caracas, p. 47-67 [repris : in Daniel R. Gross (dir.), Peoples and Cultures of Native South America. An Anthropological Reader, American Museum of Natural Story, New York, 1973, p. 98-125 ; in Patricia J. Lyon (dir.), Native South Americans. Ethnology of the Least Known Continent, Little, Brown, Boston, 1974, p. 122-132 ; in Yehudi A. Cohen (dir.), Man in Adaptation. The Cultural Present, Aldine Publishing Company in Environmental Anthropology, Chicago, 1974, p. 131-145 et in Michael R. Dove et Carol Carpenter (dir.), A Historical Reader, Blackwell Publishing, Malden (MA), 2008, p. 249-253]. 
Robert Leonard Carneiro (1927-2020)

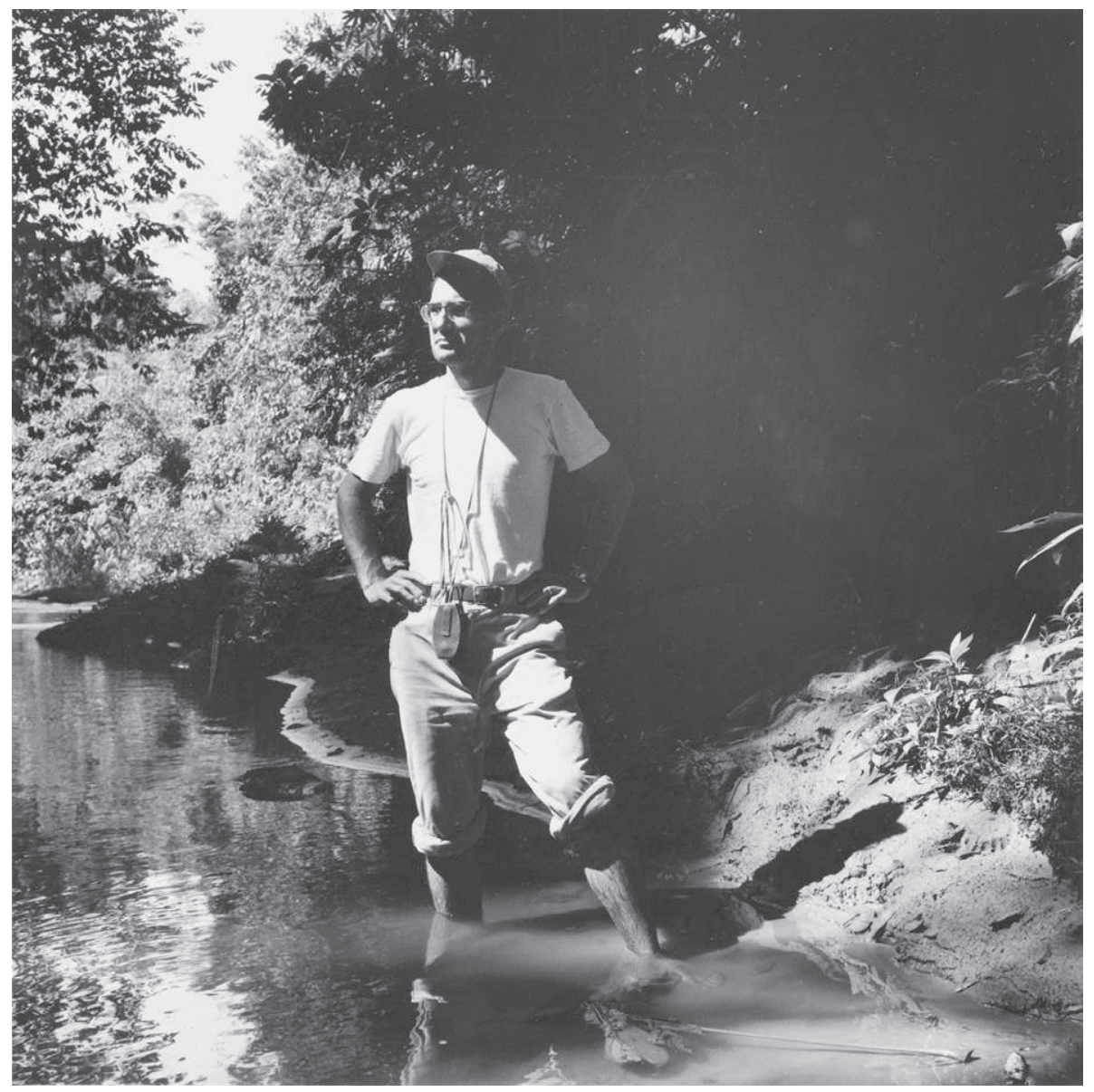

Fig. 3 - Robert Leonard Carneiro

(C) Courtesy of the Division of Anthropology, American Museum of Natural History) 
CARNEIRo Robert Leonard

1970 «A theory of the origin of the state », Science, 169, p. 733-738.

1976 « The afterworld of the Kuikuru Indians », in Ronald K. Wetherington (dir.), Colloquia in anthropology, Fort Burgwin Research Center, Dallas, vol. 1, p. 3-15.

1978 Commentaire sur « Food Taboos, Diet, and Hunting Strategy. The Adaptation to Animals in Amazon Cultural Ecology, by Eric B. Ross », Current Anthropology, 19 (1), p. 19-20.

1989 «To the village of the jaguars: the master myth of the upper Xingú », Antropológica, 72, p. 3-40.

1995 «Godzilla meets new age anthropology: facing the post-modernist challenge to a science of culture », Europaea (Italy), 1, p. 3-22.

2004 «Can anthropology be made a science? A retrospective glance », Ethnos (Sweden), 69, p. 268-288.

2009 " "The Sons of the Moon": the Amahuaca version of a widespread Amazonian myth », Amazônica-Revista de Antropologia, 1 (1), p. 54-67.

2012 «The circumscription theory: a clarification, amplification, and reformulation », Social Evolution \& History, 11 (2), p. 5-30.

2019 The Making of an Exhibit Hall. Bringing to Life Amazonian Indian Culture, AuthorHouse, Bloomington.

CARnEIRo Robert Leonard et Joyce Marcus

2006 «Craig Morris », Anthropology News, 47 (8), p. 29.

Chernela Janet

2005 «Gertrude Dole (1915-2001)», Tipití. Journal of the Society for the Anthropology of Lowland South America [en ligne], 3 (1), Article 9, https:// digitalcommons.trinity.edu/tipiti/vol3/iss1/9, consultée le 15/12/2020.

Dole Gertrude E.

1960 « Techniques of preparing manioc flour as a key to culture history in Tropical America », in Anthony F. C. Wallace (dir.), Men and Cultures. Selected Papers of the Fifth International Congress of Anthropological and Ethnological Sciences, University of Pennsylvania Press, Philadelphia, p. 241-248.

ERIKSON Philippe

1990 «Near beer of the Amazon », Natural History, 8 (90), p. 52-61.

Hewlett Christopher

2013 History, Kinship, and Comunidad. Learning to Live Together amongst Amahuaca People on the Inuya River in the Peruvian Amazon, thèse de doctorat, anthropologie sociale, University of Saint-Andrews.

2016 «Engaged anthropology grant: Christopher Hewlett» [en ligne], The WennerGren blog, http://blog.wennergren.org/2016/10/, consultée le 15/12/2020.

HuXLey Francis

1956 Affable Savages. An Anthropologist among the Urubu Indians of Brazil, Rupert Hart-Davis, London.

Huxley Matthew et Cornell CAPA

1964 Farewell to Eden, Harper \& Row, New York. 
MAuzé Marie et Joëlle RostKowski

2004 «A New Kid on the Block. Le National Museum of the American Indian », Journal de la Société des américanistes, 90 (2), p. 115-128 (http://journals. openedition.org/jsa/1465, consultée le 15/12/2020).

RuSSELl Robert

1962 « Answers to questions » [Asked by Carneiro], Información de Campo, n 23 [26 p. ; $1 \mathrm{mf}$.$] .$

SAHLINS Marshall

1976 [1974] Âge de pierre, âge d'abondance. L'économie des sociétés primitives [traduction de: Stone Age Economics], Gallimard, Paris.

Stoll David

2020 «Alumni News. Gertrude Evelyn Dole (‘37) -Midd’s first grad to earn an anthropology $\mathrm{PhD}$ was a well-known Amazonist », Field Notes. A Newsletter of the Middlebury College Anthropology Department, 15, p. 23-26.

Thomas David Hurst

2007 «Edward Craig Morris (1939-2006)», American Anthropologist, 109 (4), p. 789-792.

TschopIK Harry

1952 Indians of the Montaña, Man and Nature Publications of the American Museum of Natural History (Science Guide, 135), New York.

URTON Gary

1992 «The hall of South American peoples. Permanent installation at the American Museum of Natural History, New York, NY », Museum Anthropology, 16 (2), p. 46-48. 
